\title{
CARACTERIZAÇÃO REOLÓGICA DA POLPA DE ARATICUM (ANNONA CRASSIFLORA MART)
}

\author{
C. G. RODRIGUES ${ }^{1}$, R. C. C. DOMINGUES ${ }^{1}$, W. A. SILVA. ${ }^{1}$, M. H. M. REIS ${ }^{2}$ L. A. \\ CARLOS $^{1}$ e F. C. CALLEGARI ${ }^{3}$ \\ ${ }^{1}$ Universidade Federal de São João Del-Rei - Departamento de Engenharia de Alimentos \\ ${ }^{2}$ Universidade Federal de Uberlândia - Faculdade de Engenharia Química \\ ${ }^{3}$ Universidade Federal de Minas Gerais - Departamento de Engenharia Química \\ E-mail para contato: ruidomingues@ufsj.edu.br
}

RESUMO - O objetivo deste trabalho foi avaliar a reologia da polpa filtrada de Marolo (Annoma crassiflora) variando-se a temperatura entre 10 e $40^{\circ} \mathrm{C}$, utilizando-se os modelos reológicos de Bingham, Herschel-Bulkley e Ostwald-de-Waele para o ajuste dos dados experimentais. Foi utilizado um viscosímetro Brookfield LVDV-II + PRO com configuração de cilindros concêntricos. Os modelos reológicos foram analisados utilizando o software Statistica 7, sendo os parâmetros dos modelos nãolineares estimados pelo método de Gauss-Newton. A polpa de marolo filtrada apresentou caráter pseudo-plástico. $O$ modelo que melhor representou os dados experimentais foi o modelo de Herschel-Bulkley, apresentando índices de fluidez na faixa de $\mathrm{n}=0,73$, índices de consistência na faixa de $79,79 \mathrm{mPa} \cdot \mathrm{s}$, e ausência significativa de tensão residual par ao escoamento. Não foi observada influência significativa das viscosidades aparentes com o aumento da temperatura.

\section{INTRODUÇÃO}

Dentre vários países produtores de frutas, o Brasil é considerado o terceiro maior, com produção em torno de 34 milhões de toneladas, numa área de 2,2 milhões de hectares, proporcionando 4 milhões de emprego e PIB agrícola de US\$ 11 bilhões. (SIMON, 2001). De acordo com a FAO (Food ad Agriculture Organization, 2014) o comércio de frutas atingiu, em 2000, algo em torno de 10\%, da produção mundial de frutas e hortaliças, com tendência de crescimento em função da preferência dos consumidores por frutas e vegetais frescos.

O bioma do Cerrado é o maior bioma brasileiro, apresenta diversos ecossistemas e diversificada flora, com mais de 12000 espécies de plantas. Cerca de 110 espécies de plantas possuem potencial econômico, incluindo fruteiras, palmeiras, madeiras, medicinais, condimentares e oleaginosas. Apesar de tanta riqueza, suas frutíferas tem sido pouco utilizadas. Seus frutos apresentam sabores característicos, proteínas, sais minerais, ácidos graxos, vitaminas do complexo B e carotenóides, além de atrativos sensoriais como cor, sabor, aroma peculiares e intensos. Entretanto são pouco explorados comercialmente. (SILVA et al., 2001). 
Dentre os frutos do cerrado, o Marolo, também conhecido por araticum, panã ou cabeça de nego (Annoma crassiflora Mar), representa bem as características descritas, como por exemplo, composição rica em vitaminas além de ferro, fósforo e cálcio (ALMEIDA, 1998). Sua polpa é consumida por populações locais em sua grande maioria in natura ou em forma de sorvetes, sucos ou geleias (SILVA et al., 2001); de aparência arenosa, disposta em vários gomos amarelados contendo sementes em cada um, de sabor e aroma característicos. (Figura $1)$.

$\mathrm{Na}$ indústria de transformação de alimentos, utiliza-se a polpa da fruta que será submetida a etapas durante o processamento, como por exemplo agitação, bombeamento e transporte por tubulações. Para que essas etapas sejam economicamente otimizadas, é de fundamental importância conhecimentos de propriedades físicas e químicas da polpa submetida a tais processos. Uma dessas propriedades é o comportamento reológico, que ocupa uma posição de destaque, tornando-se útil além da medida de qualidade, como também em execução de projetos, aquisição, avaliação e operação de equipamentos processadores de alimentos tais como as bombas, sistemas de agitação, tubulações (IBARZ et al, 1996).

Neste sentido, o objetivo do presente trabalho consiste na caracterização reológica da polpa de Marolo, efetuada no Laboratório de Ciência e Tecnologia de Alimentos I, da UFSJ/ Campus Sete Lagoas MG.

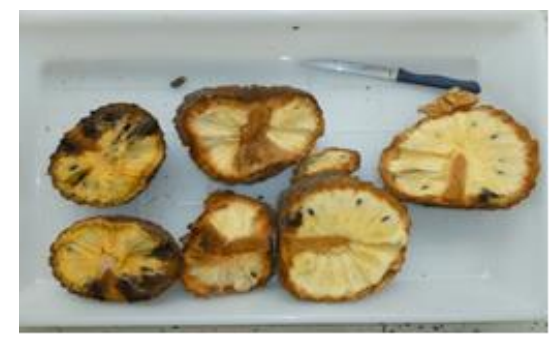

Figura 1- Marolo, corte transversal. Fonte Arquivo Pessoal

\section{MATERIAIS E MÉTODOS}

Foi utilizada como matéria-prima polpa de Marolo (Annona crassiflora Mart.) adquirida na região de Sete lagoas - MG. A polpa foi previamente filtrada usando tecido malha fina, do tipo "amorim" e mantida congelada a $-18^{\circ} \mathrm{C}$ e descongelada até temperatura ambiente para utilização nos experimentos.

As viscosidades foram analisadas com o auxílio de um viscosímetro digital Brookfield LVDV-II+ PRO, com geometria de cilindros concêntricos. Um banho ultratermostatizado foi utilizado para o controle da temperatura das amostras no decorrer dos experimentos. As viscosidades foram analisadas às temperaturas de $10,15,20,25,30,35$ e $40^{\circ} \mathrm{C}$. Foi utilizado um adaptador que acoplado ao banho ultratermostatizado permitiu o controle da temperatura durante as análises (Figura 2) sendo que para cada temperatura analisada a rotação do spindle foi variada buscando aplicar o maior espectro de taxas de deformação possível. Foi verificado que essa faixa foi de 0 a $14,72 s^{-1}$ para todas as amostras. 

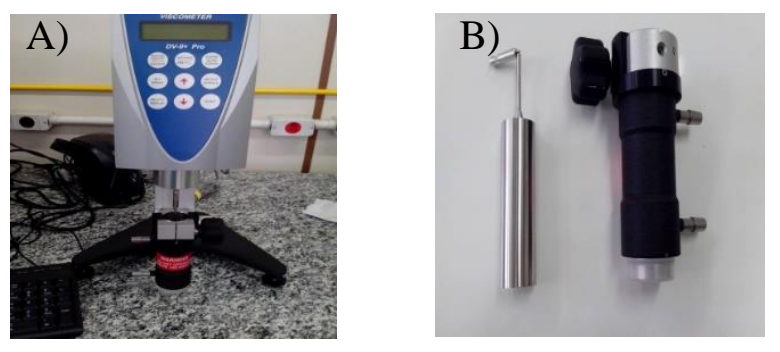

Figura 2- A) Viscosimetro, B) Spindle e adaptador de amostra.

Fonte Arquivo Pessoal

\subsection{Cálculo dos parâmetros dos modelos reológicos}

Os valores experimentais obtidos em relação a tensão de cisalhamento e taxa de deformação foram ajustados, através de regressão não linear, aos modelos de reológicos de Bingham (Equação 1), Herschel-Bulkley (Equação 2) e Ostwald-de-Waele (Equação 3).

$$
\begin{aligned}
& \tau=K_{0, B}+K_{B}(\dot{\gamma}) \\
& \tau=K_{0, H B}+K_{H B}(\dot{\gamma})^{n_{H B}} \\
& \tau=K_{o w}(\dot{\gamma})^{n_{o w}}
\end{aligned}
$$

Onde $\tau$ é a tensão de cisalhamento $(\mathrm{Pa}), \dot{\gamma}$ é a taxa de deformação $\left(s^{-1}\right), \mathrm{K}_{\mathrm{B}}, \mathrm{K}_{\mathrm{HB}}, \mathrm{K}_{\mathrm{OW}}$ são os índices de consistência $(\mathrm{mPa} \cdot \mathrm{s}), \mathrm{K}_{0, \mathrm{~B}}$ e $\mathrm{K}_{0, \mathrm{HB}}(\mathrm{Pa})$ são as tensões residuais dos respectivos modelos. Os parâmetros $\eta_{B}, n_{H B}$ e $\eta_{0 W}$ (adimensionais) são os índices de fluxo.

Os parâmetros reológicos foram determinados a partir do ajuste dos dados experimentais de tensão de cisalhamento em função da taxa de deformação com cada equação apresentada. Este ajuste foi realizado com o auxílio do software Statistica 7.0 (Statsoft) utilizando o método de Gauss-Newton.

$\mathrm{O}$ efeito da temperatura sobre a viscosidade aparente foi descrito mediante uma equação análoga à de Arrhenius (IBARZ et al., 2003), conforme apresentado na Equação (4).

$$
\eta_{a}=\eta_{\infty} \exp \left(\frac{E_{a}}{R T}\right)
$$

Onde $\eta_{a}$ é a viscosidade aparente $(m P a \cdot s) \eta_{\infty}$ é a viscosidade à deformação infinita, $\mathrm{R}$ é a constante universal dos gases, $E_{a}$ é a energia de ativação para o escoamento e T $(\mathrm{K})$ a temperatura.

A Equação (4) indica a tendência geral observada de uma diminuição da viscosidade aparente com o aumento da temperatura. De um modo geral, quanto maior for a energia de ativação, maior será o efeito da temperatura sobre a viscosidade (SILVA et al., 2005). 
A temperatura pode afetar diferentes parâmetros reológicos como a viscosidade, índice de consistência e tensão residual. Usualmente decréscimo na viscosidade e índices de consistência são observados com o aumento da temperatura (IBARZ et al., 2003).

\section{RESULTADOS E DISCUSSÕES}

A Figura 3 apresenta os resultados obtidos de tensão de cisalhamento em função da taxa de deformação para polpa de Marolo filtrada nas temperaturas avaliadas:

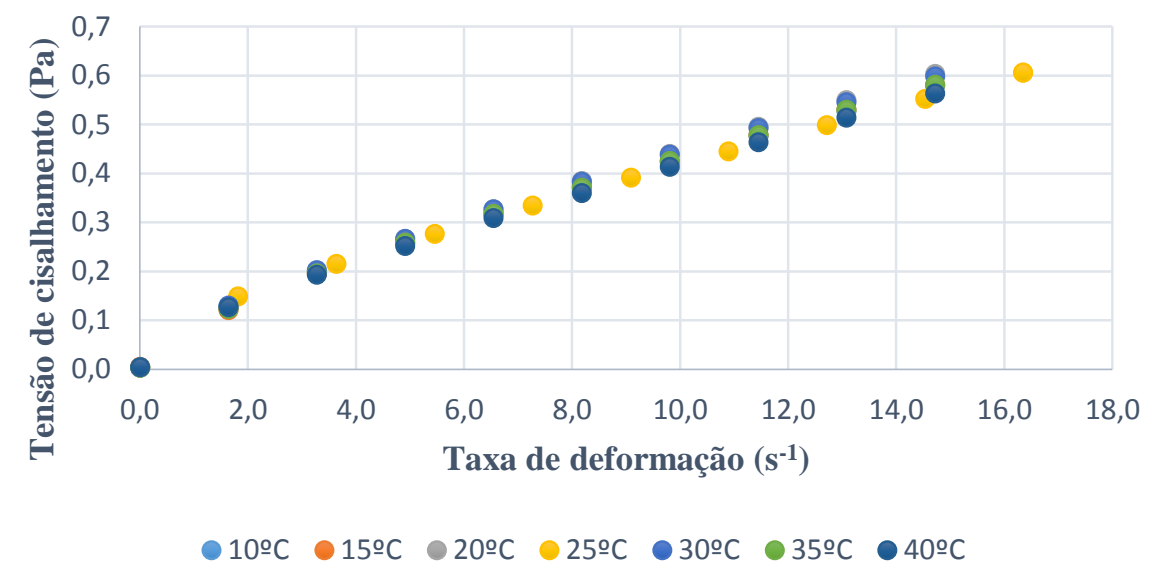

Figura 3 - Tensão de cisalhamento em função da taxa de deformação para polpa filtrada de Marolo

$\mathrm{Na}$ Figura 3 não é observada variação aparente da viscosidade das amostras com a temperatura. Este comportamento pode ser também notado na Figura 4:

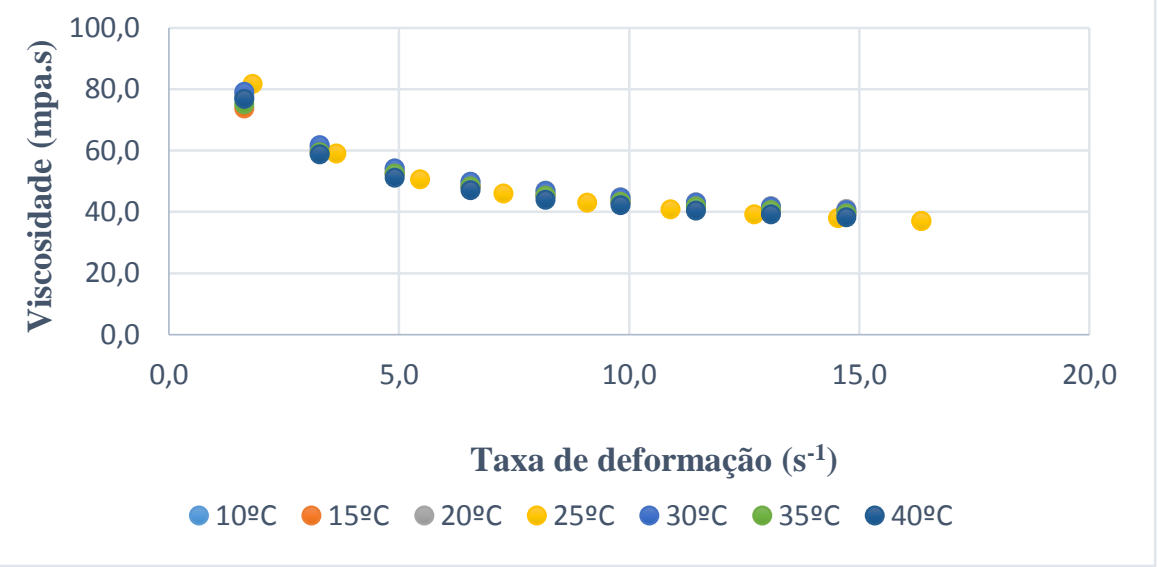

Figura 4 - Viscosidade aparente em função da taxa de deformação para polpa filtrada de Marolo 
Em uma análise preliminar, a Figura 3 apresenta um comportamento de aparente linearidade entre a taxa de deformação e a tensão de cisalhamento para todas as amostras, indicando comportamento Newtoniano. Entretanto, observando a Figura 4, percebe-se que a viscosidade aparente diminui a taxa de deformação aplicada, o que sugere comportamento pseudo-plástico. A modelagem matemática dos dados de tensão de cisalhamento em função da taxa de deformação, conforme apresentado na Tabela 1, confirma as características das curvas.

Tabela 1Parâmetros Reológicos para polpa de Marolo

\begin{tabular}{ccccccccccc} 
Modelo & Parâmetro & Unidade & \multicolumn{7}{c}{ Temperatura $\left({ }^{\circ} \mathbf{C}\right)$} \\
\hline \multirow{3}{*}{ Bingham } & & & 10 & 15 & 20 & 25 & 30 & 35 & 40 \\
& $K_{0, B}$ & $N \cdot m^{-2}$ & 0,057 & 0,055 & 0,056 & 0,068 & 0,058 & 0,055 & 0,057 \\
& $K_{B}$ & $m P a \cdot s$ & 36,80 & 37,20 & 38,60 & 33,70 & 38,10 & 37,20 & 35,80 \\
& $\mathrm{R}^{2}$ & & 0,906 & 0,01 & 0,855 & 0,414 & 0,531 & 0,813 & 0,494 \\
\hline \multirow{3}{*}{ Ostwald-de-Waele } & $K_{O W}$ & $m P a \cdot s$ & 82,90 & 96,00 & 84,60 & 93,10 & 89,90 & 82,50 & 86,60 \\
& $n_{O W}$ & & 0,72 & 0,65 & 0,73 & 0,66 & 0,70 & 0,72 & 0,69 \\
& $\mathrm{R}^{2}$ & & 0,982 & 0,959 & 0,986 & 0,976 & 0,986 & 0,988 & 0,984 \\
\hline \multirow{3}{*}{ Herschel-Bulkley } & $K_{0, H B}$ & $N \cdot m^{-2}$ & 0,004 & 0,005 & 0,004 & 0,007 & 0,004 & 0,003 & 0,005 \\
& $K_{H B}$ & $m P a \cdot s$ & 80,80 & 77,50 & 80,70 & 80,70 & 81,80 & 79,00 & 78,00 \\
& $n_{H B}$ & & 0,73 & 0,74 & 0,74 & 0,71 & 0,73 & 0,74 & 0,73 \\
& $\mathrm{R}^{2}$ & & 0,999 & 1 & 1 & 0,999 & 1 & 1 & 0,999 \\
\hline
\end{tabular}

$\mathrm{R}^{2}$ - Coeficiente de determinação

O Modelo de Bingham pressupõe que exista uma linearidade entre a taxa de deformação e tensão de cisalhamento de um fluido e também a presença de uma tensão residual para o escoamento. Observa-se pelos resultados da Tabela 1 que o modelo de Bingham fora o que representou de forma menos satisfatória os dados experimentais, por ter apresentado os menores valores de $\mathrm{R}^{2}$ em todas as temperaturas analisadas.

Os modelos de Ostwald-de-Waele (também conhecido como Power Law) e HerschelBulkley apresentaram em todas as temperaturas valores de $\mathrm{R}^{2}$ próximos da unidade, o que indica uma boa representação dos modelos aos dados experimentais, sendo o modelo de HerschelBulkley ligeiramente mais preciso. $\mathrm{O}$ valor do parâmetro Índice de fluxo $\left(n_{H B}\right.$ e $\left.n_{O W}\right)$ indicam o grau de pseudo-plasticidade da amostra, sendo que quanto mais próximo da unidade, mais a mesma se comporta como um fluido Newtoniano. Analisando os valores do índice de fluxo a todas as temperaturas, nos modelos de Ostwald-de-Waele e Herschel-Bulkley foram observados valores médios de 0,7 (desvio médio $\pm 0,025)$ e 0,73 ( $\pm 0,01)$ respectivamente, mostrando variação desprezível dos parâmetros com a temperatura. Tendo em vista que todos os valores observados foram menores que 1, é possível se classificar a polpa filtrada de Marolo como um fluido pseudoplástico em toda a faixa de temperaturas analisada.

Os parâmetros índice de consistência $\left(K_{H B}\right.$ e $\left.K_{O W}\right)$ apresentaram Valores médios de 87,94 
$( \pm 4,33)$ e $79,79( \pm 1,39) m P a \cdot s$ para o modelo de Ostwald-de-Waele e Herschel-bulkley respectivamente. A ordem de grandeza do desvio médio dos valores também leva a conclusão de que os índices de consistência não variam na faixa de temperaturas avaliada.

O modelo de Herschel-Bulkley considera a presença da tensão residual para o escoamento, e os valores dos parâmetros $K_{0, H B}$ observados a todas as temperaturas se encontram na ordem de $10^{-3} N \cdot m^{-2}$ podendo ser desprezados. Dessa forma, admite-se que a polpa de Marolo filtrada não necessita da aplicação de uma tensão para que se inicie o escoamento.

$\mathrm{O}$ ajuste da viscosidade aparente medida na taxa de deformação de $8,18 \mathrm{~s}^{-1}$ em função da temperatura de acordo com a Equação (4) determinou valores de $\eta_{\infty}=0,449 \mathrm{mPa} \cdot \mathrm{s}$ e $E_{a}=$ $0,543 \mathrm{~J} \cdot \mathrm{mol}^{-1}$. O baixo valor da energia de ativação encontrado confirma a baixa dependência da viscosidade aparente com a temperatura.

O comportamento pseudo-plástico é um comportamento comum em polpas de frutas, sendo o mesmo amplamente reportado na literatura para sucos e polpas de frutas. Segundo Holdsworth (1971) e Ibarz et al (2003) a maioria dos alimentos fluidos apresenta comportamento pseudoplástico, sendo a diminuição da viscosidade aparente com o aumento da taxa de deformação explicada pelo fato de que sob o efeito de maiores tensões moléculas de cadeia longa tendem a se desembaraçar diminuindo a resistência intermolecular ao escoamento.

\title{
4. CONCLUSÕES
}

Observou-se que o aumento da temperatura, de 15 a $40^{\circ} \mathrm{C}$, não provocou alterações significativas na viscosidade aparente da polpa de Marolo filtrada. Em todas as temperaturas analisadas, a polpa de Marolo filtrada apresentou comportamento não-Newtoniano, com característica pseudo-plástica e sem presença de tensão residual. Os modelos que melhor representaram o comportamento reológico foram os modelos de Herschel-Bulckey e Ostwald-deWaele, apresentando parâmetros similares de índice de consistência e Índice de fluxo.

\section{AGRADECIMENTOS}

Os autores agradecem à FAPEMIG e a PROPE-UFSJ pelo apoio financeiro.

\section{REFERÊNCIAS}

\author{
-ALMEIDA, S. P.; PROENÇA, C. E. B.; SANO, S. M.; RIBEIRO, J. F. Cerrado: espécies \\ vegetais úteis. Empresa Brasileira de Pesquisa Agropecuária do Brasil (EMBRAPA), Brasil, \\ 1998 , \\ p. 48-335.
}

-FAO - FOOD AND AGRICULTURE ORGANIZATION. Disponível em: $<$ http://apps.fao.org/cgibin/nph-db.pl?subset=agriculture> Acesso em : 20. abril. 2014. 
-HOLDSWORTH, S. D. Aplicability of rheological models to the interpretation of low and processing behavior of fluid products. Journal of Texture Studies, v.2, n.4, p. 393-418, 1971.

-IBARZ, A.; Barbosa-Cànovas, G. V. Unit operations in food engineering. Boca Raton: CRC Press, 2003. 889 p.

-IBARZ, A.; GONÇALVES, C.; EXPLUGAS, S. Rheology of Clarified Passion Fruit Juices. Fruit Processing, v.6, n.8, p.330-333, 1996.

-PELEGRINE, D. H.; Silva, F. C.; Gasparetto, C. A. (2002). Rheological Behavior of Pineapple and Mango Pulps. LWT - Food Science and Technology, 35, 645-648.

-SILVA, D. B.; SILVA, J. A.; JUNQUEIRA, N. T. V.; ANDRADE, L. R. M. 2001. Frutas nativas

dos cerrados. Brasília: EMBRAPA-CPAC, 179 p.

-SILVA, F. C.; Guimarães, D. H. P.; Gasparetto, C. A. (2005). Rheology of acerola juice: effects of concentration and temperature. Ciência Tecnologia Alimentos 25, 121-126.

-SIMON, C.W. A importância da fruticultura no contexto do agribusiness. In: ENFRUTE, IV, 2001, Fraiburgo. Anais do IV Encontro Nacional sobre Fruticultura de Clima

Temperado, Fraiburgo, 2001. 